\title{
EFFECTS OF A HARMONIC CAVITY AT THE ESRF
}

\author{
J. Jacob, O. Naumann, W. Beinhauer, ESRF, Grenoble, France
}

\section{Abstract}

The installation of a harmonic cavity to provide bunch lengthening and thereby increase the Touschek lifetime is considered for the ESRF storage ring. Simulations with a particle tracking code have shown that for few bunch operation - where the current per bunch is large - a strong lengthening already results from the coupling with the broad band impedance of the vacuum chamber. Therefore the net gain with an additional harmonic cavity is only moderate. For multibunch operation, however, where the current per bunch is small, a harmonic cavity is expected to increase the bunch length by up to a factor six, with a corresponding increase in Touschek lifetime. The Landau damping provided by the additional nonlinearity of the RF voltage has also been studied in detail. As an important and somewhat surprising result, it turns out that the thresholds for longitudinal coupled bunch instabilities may even be reduced when the operation parameters of the harmonic cavity are optimised to provide a maximum bunch lengthening.

\section{INTRODUCTION}

The installation of more and more narrow gap insertion devices at the ESRF has increased the transverse impedance of the storage ring. Since this has lowered the head-tail stability limits, higher chromaticities have to be used as a remedy. This, however, reduces the dynamic aperture and the energy acceptance, with the further consequence of a lower Touschek lifetime, especially in single and few bunch filling, where the current per bunch is high. To optimise the Touschek lifetime in few bunch operation, the acceleration voltage used to date at the ESRF is around $8 \mathrm{MV}$ instead of the possible $12 \mathrm{MV}$ : this maximises the bunch length without penalising the energy acceptance. Lengthening the bunches also has the beneficial side effect of an increased head-tail stability limit, since the overlap of the beam spectrum with the broadband impedance is smaller for longer bunches.

A precise control of the bunch length without reduction of the RF-acceptance should be possible with a higher harmonic cavity. This paper summarises the possible performance upgrade with such a cavity for the ESRF storage ring. Section 2 describes the achievable bunch lengthening as a function of the harmonic number $n$ and a deviation from the optimum working point. The expected gain in bunch length for single and few bunch operation is presented in section 3 , taking into consideration the already strong effect from the impedance of the storage ring. The results from a detailed analysis of the effect on longitudinal coupled bunch instabilities, taking into account Landau damping which results from the non-linearity in the RF-voltage, are given in section 4.

\section{BUNCH LENGTHENING WITH A HARMONIC CAVITY}

A double RF-system allows to shape the accelerating voltage at the time of passage of the synchronous particle [1]. Both the relative phase and the harmonic voltage level $V_{H}$ can be used to adjust the harmonic system. Two conditions allow the determination of the operation values for optimum bunch lengthening: firstly, for maximum bunch length the slope of the accelerating voltage has to be horizontal at the bunch centre and, secondly, the accelerating voltage should not be curved at this point, to avoid the forming of micro-bunches. In order to maintain the same time of synchronous passage, it is additionally necessary to adjust the amplitude of the normal voltage by a factor slightly different from unity. All these quantities can be expressed in terms of the time of synchronous passage and the harmonic number $n$.

Fig. 1 shows how the zero current bunch length varies in the case of the ESRF as a function of the harmonic voltage level $V_{H}$. Since the maximum bunch lengthening factor, of approximately 6 for $n=3$, is reached only very close to the optimum value $V_{O P T}$ for $V_{H}$, finding and maintaining a good working point is likely to become a delicate operation as very precise control of the cavity parameters is necessary.

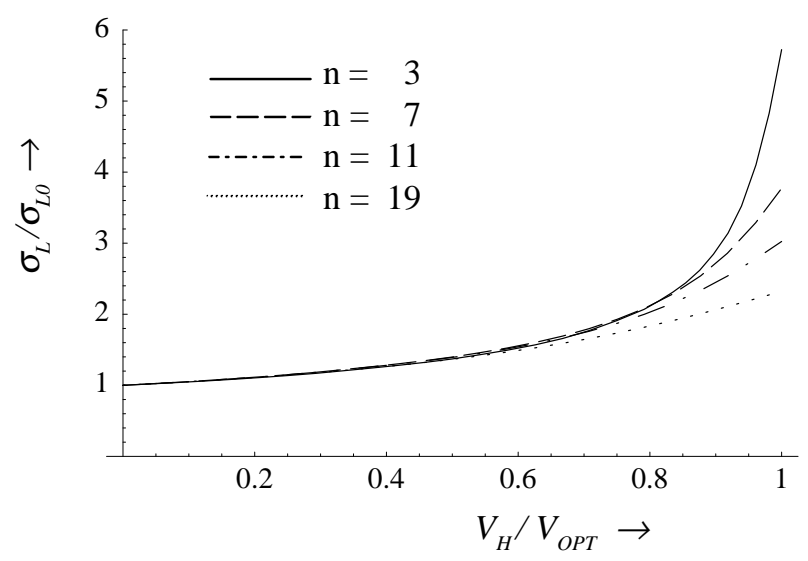

Figure 1: Bunch length $\sigma_{L}$ as a function of the harmonic voltage level $\left(\sigma_{L O}\right.$ : bunch length for $I_{b}=0$ and $\left.V_{H}=0\right)$

Fig. 1 also shows how a variation of the harmonic number $n$ influences the dependency of the bunch 
lengthening factor on the harmonic voltage. Although values of $n>7$ are of merely academic interest, the comparison shows how the choice of a higher value for the harmonic number $n$ reduces the sensibility on the harmonic cavity parameters. This means easier operation conditions, but limits the maximum possible increase in bunch length.

\section{PARTICLE TRACKING FOR HIGH CURRENT PER BUNCH}

With increasing current per bunch, several effects occur. Firstly, transverse head-tail instabilities have to be mastered by operating the storage ring with an increased chromaticity, which unfortunately also reduces the dynamic energy acceptance and the associated lifetime. Secondly, the higher charge density increases the Touschek effect, leading to an overall lifetime of the order of 4 hours in single bunch operation at $15 \mathrm{~mA}$ and about 9 hours in 16 bunch operation at $90 \mathrm{~mA}$. This already takes into account the high natural bunch lengthening due to the interaction with the broadband impedance of the ring, as well as the choice of a rather low accelerating voltage of $8 \mathrm{MV}$, as a compromise between a maximum bunch length and sufficient RFacceptance.

The gain in bunch length that could be obtained with a third harmonic cavity has been evaluated by adapting a multiple particle tracking code. It solves the synchrotron equation turn by turn, taking into account the broadband resonator model BBR ( $Q=1, R=42 \mathrm{k} \Omega, f r=30 \mathrm{GHz})$ of the ESRF storage ring [2]. For the present study, the code has been modified to incorporate the harmonic voltage distortion, assuming an optimum amplitude and phase of the modulating voltage.

Table 1

\begin{tabular}{|l|c|c|c|}
\hline Current per bunch & $\mathbf{1 0} \mathbf{~ m A}$ & $\mathbf{1 5} \mathbf{~ m A}$ & $\mathbf{2 5} \mathbf{~ m A}$ \\
\hline $\begin{array}{l}\text { Lengthening factor } \\
\text { from BBR only }\end{array}$ & 3.6 & 4.2 & 5.4 \\
\hline $\begin{array}{l}\text { Lengthening factor from } \\
\text { BBR and 3 }{ }^{\text {rd }} \text { harmonic cavity }\end{array}$ & 6.2 & 6.6 & 7.6 \\
\hline $\begin{array}{l}\text { Net gain in bunch length with } \\
\text { a harmonic cavity }\end{array}$ & $+72 \%$ & $+57 \%$ & $+41 \%$ \\
\hline
\end{tabular}

In the limit of zero current, the simulations confirmed the theoretical bunch lengthening by a factor 6 for $n=3$ in Fig. 1. They also confirmed that the energy spread is only affected by the BBR and not influenced by the presence of a harmonic cavity. As shown in table 1 , for the nominal single bunch current of $15 \mathrm{~mA}$, the interaction with the BBR already lengthens the bunch by a factor 4.2 , so that a harmonic cavity would at best give $57 \%$ additional lengthening.

If a passive cavity is to be used, it must exhibit a shunt resistance of the order of several $100 \mathrm{M} \Omega$ in order to provide the optimum harmonic voltage with a driving current of about $10 \mathrm{~mA}$. The only feasible options would therefore be either a passive superconducting or an active normal conducting cavity. The final choice, however, needs further investigations.

\section{EFFECT ON MULTIBUNCH OPERATION}

The effect of main interest in the application for multi-bunch operation is the increase of the Touschek lifetime, that accompanies the increased bunch length, especially in the case where the effect of an increased energy acceptance has an upper limit, as is the case at the ESRF. In multi-bunch operation the per-bunch current intensity is small (of the order of tenths of $\mathrm{mA}$ ), therefore single-bunch effects such as the head-tail instability play only a minor role. Still, the additional bunch lengthening should allow a further reduction of the chromaticity.

As far as the effect on multi-bunch instabilities is concerned, it is commonly assumed that the spread in synchrotron frequencies within a bunch due to the distorted RF-voltage leads to increased stability limits because of the additional Landau damping. A closer investigation, however, reveals that this need not necessarily be the case.

\subsection{Effect on the synchrotron frequency density}

The density $\rho$ of the synchrotron frequency $f_{s}$ can be calculated using the formula

$$
\rho\left(f_{s}\right)=-T^{2}(H) \psi(H)\left(\frac{d A}{d H}\right) /\left(\frac{d T}{d H}\right)
$$

where $A$ and $T$ are the quantities defined on page 3 of [1]. Inverting the relation $T(H)$ gives a function in $f_{s}=$ $1 / T(H)$.

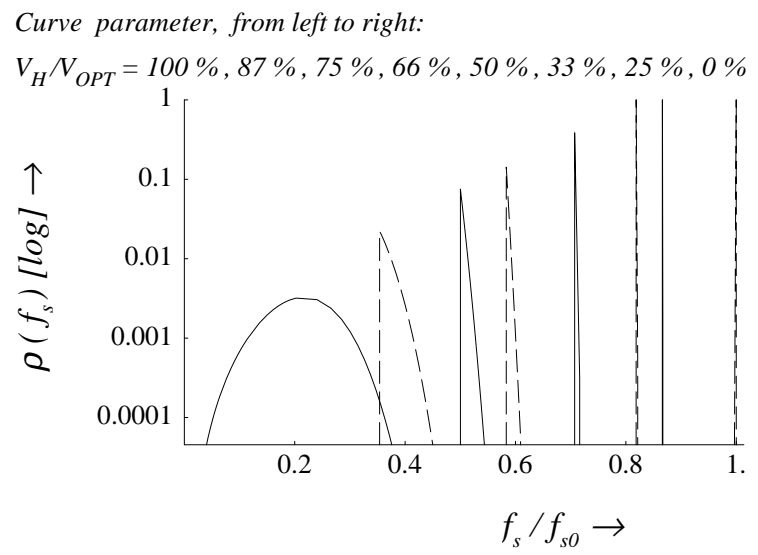

Figure 2: Synchrotron frequency distributions for various levels of harmonic voltage and $n=3$

Fig. 2 shows a sequence of densities for the case $n=3$. The natural frequency density spread for $V_{H}=0$ is extremely small, because the non-linearity is weak. With 
a change in operation parameters, the centre of the distribution is shifted towards smaller frequencies and the spread increases, although only considerably so close to the optimum choice of operation parameters. This can be explained from the fact, that - especially for a small harmonic number - the accelerating voltage is distorted on a scale much larger than the bunch. Thus, switching on the modulation changes the voltage slope for all particles in almost the same way, the bunch being too short to profit from the additional non-linearity. Consequently the synchrotron frequency density is not spread out, but is shifted in the mean because of the overall reduced slope. It is only as the optimum voltage is approached, that the bunch becomes large enough for some particles to reach the region of increased nonlinearity, which then increases the frequency spread.

\subsection{Dispersion relation and threshold current}

In order to determine the net effect on longitudinal multi-bunch instability thresholds due to cavity higher order modes (HOM), a dispersion relation has to be solved. The values entering into it are the impedance $Z_{\text {Hом }}$ and the resonance frequency $f_{\text {НОм }}$ of the HOM and several machine parameters: $\alpha$ (momentum compaction), $E_{0}$ (energy), $T_{0}$ (revolution time), and $\delta_{n}$ (natural radiation damping).

$$
1=j \frac{\alpha 2 \pi f_{H O M} Z_{H O M} I_{b}}{T_{0} E_{0} / e} \int \frac{\rho\left(\omega_{s}^{\prime}\right) d \omega_{s}^{\prime}}{\omega_{s}^{\prime 2}-\omega^{2}+2 j \delta_{n} \omega}
$$

This has to be solved for the real coherent synchrotron frequency $\omega$ and the beam current $I_{b}$ in order to determine the threshold current $I_{t h}[3]$.

The threshold current $I_{t h}$ can be plotted as a function of $V_{H}$ by applying this formula, as is shown in Fig. 3. Increasing $V_{H}$, one first observes a decrease of $I_{t h}$, up to a turning point, which is reached earlier the larger $n$ is, but in any case a valley of decreased stability always has to be crossed.

\subsection{Application to the ESRF}

Investigating the values for the optimum operation parameters that can be seen on Fig 3, we discover that a value of $n>7$ would at least allow to break even as far as thresholds are concerned. However, in order to provide real protection against instabilities, a much higher value would be necessary. Then, of course, one has to pose the question of how to construct a harmonic cavity with a high $n$. Such a choice would also be discouraged as the optimum gain in bunch length would be much smaller (Fig. 1).

Therefore, one important result is that for the practical choices of $n=3$ or 5 , additional means to damp or tune HOMs away from synchrotron sidebands are necessary, if a harmonic cavity is not to seriously compromise the multi-bunch instability thresholds. At the ESRF, this can be achieved with the recently commissioned highly accurate temperature control system for the accelerating cavities (error $<0.1^{\circ} \mathrm{C}$ ).

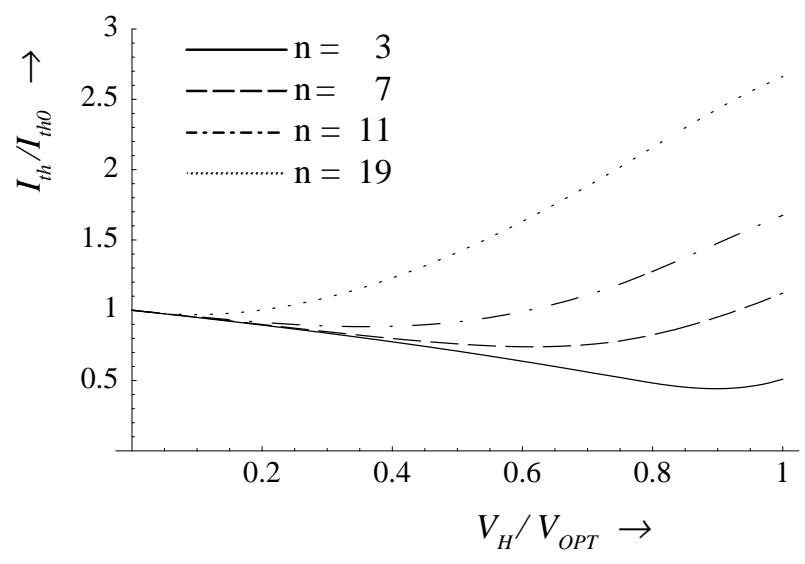

Figure 3: Multibunch instability threshold $I_{t h}$ as a function of the harmonic voltage $\left(I_{t h 0}\right.$ : value for $\left.V_{H}=0\right)$

An interesting alternative would be to operate the harmonic system at a frequency of a multiple of the RFfrequency plus the revolution frequency ("harmonic plus one" cavity). This modulates the RF-voltage around the circumference of the storage ring and provides a bunchto-bunch frequency spread, which has a much stronger Landau damping effect [4]. This, however, still requires some investigation.

\section{SUMMARY AND CONCLUSIONS}

A harmonic cavity at the ESRF would provide only a limited net increase of the bunch length for single and few bunch operation, because of the already strong lengthening due to the broadband impedance. For standard multi-bunch operation, however, the net increase can be much larger. The somewhat higher sensitivity to multi-bunch instabilities can easily be overcome by means of the cavity temperature control.

\section{REFERENCES}

[1]A. Hofmann and S. Myers, "Beam Dynamics in a double RF System", In W.S. Newman, editor, Proc. Of the $11^{\text {th }}$ International Conference on High Energy Accelerators, pp 610-614, Boston, (1980)

[2]G. Besnier, T.F.Günzel, J.-L.Laclare, C.Limborg, "Simulation of the Evolution of a Bunch due to the Machine Impedance", $10^{\text {th }}$ ICFA Beam Dynamics Panel Workshop on 4th Generation Light Sources, Grenoble, (1996)

[3]O. Naumann and J. Jacob, "Fractional Filling Induced Landau Damping of Longitudinal Instabilities at the ESRF", PAC'97,Vancouver, (1997)

[4]O. Naumann and J. Jacob, "Landau Damping of Longitudinal Instabilities for the Operation of the ESRF Storage Ring", EPAC'98, Stockholm, (1998) 